\title{
Polymers with antiviral properties: A brief review
}

\author{
Sławomir Zmonarski ${ }^{1,-F}$, Jakub Stojanowski ${ }^{2, A-D}$, Joanna Zmonarska ${ }^{2, D}$ \\ ${ }^{1}$ Department of Nephrology and Transplantation Medicine, Wroclaw Medical University \& University Hospital, Poland \\ ${ }^{2}$ Students' Scientific Club, Department of Nephrology and Transplantation Medicine, Wroclaw Medical University, Poland \\ A - research concept and design; $\mathrm{B}$ - collection and/or assembly of data; $\mathrm{C}$ - data analysis and interpretation; \\ $\mathrm{D}$ - writing the article; $\mathrm{E}$ - critical revision of the article; $\mathrm{F}$ - final approval of the article
}

\section{Address for correspondence}

Sławomir Zmonarski

E-mail: slawomir.zmonarski@umed.wroc.pl

\section{Funding sources}

Scientific Program No. ST.C160.17.021

of the Wroclaw Medical University, Poland.

\section{Conflict of interest}

None declared

\section{Acknowledgements}

The authors would like to thank prof. Magdalena Krajewska from the Wroclaw Medical University for her support in writing this work.

Received on December 1, 2020

Reviewed on December 10, 2020

Accepted on December 13, 2020

\begin{abstract}
Viruses that are pathogenic to humans and livestock pose a serious epidemiological threat and challenge the world's population. The SARS-COV-2/COVID-19 pandemic has made the world aware of the scale of the threat. The surfaces of various materials can be a source of viruses that remain temporarily contagious in the environment. Few polymers have antiviral effects that reduce infectivity or the presence of a virus in the human environment. Some of the effects are due to certain physical properties, e.g., high hydrophobicity. Other materials owe their antiviral activity to a modified physicochemical structure favoring the action on specific virus receptors or on their biochemistry. Current research areas include: gluten, polyvinylidene fluoride, polyimide, polylactic acid, graphene oxide, and polyurethane bound to copper oxide. The future belongs to multi-component mixtures or very thin multilayer systems. The rational direction of research work is the search for materials with a balanced specificity in relation to the most dangerous viruses and universality in relation to other viruses.
\end{abstract}

Key words: COVID-19, invasive virions, virucidal properties, antiviral polymers, heavy metal particles

Cite as

Zmonarski S, Stojanowski J, Zmonarska J.

Polymers with antiviral properties: A brief review.

Polim Med. 2020;50(2):79-82. doi:10.17219/pim/131643

DOI

10.17219/pim/131643

\section{Copyright}

(C) 2020 by Wroclaw Medical University This is an article distributed under the terms of the Creative Commons Attribution 3.0 Unported (CC BY 3.0) (https://creativecommons.org/licenses/by/3.0/) 


\section{Introduction}

Human epithelial cells of the nose and throat cavity express high abundance of the receptor ACE2 (ACE-R) utilized for high-affinity virus binding and neurolipin-1, which also aids viral invasion. Both create vulnerable environment for the SARS-CoV-2/COVID-19 entry. In addition to its strong binding to ACE2-R, the greater infectivity of SARS-CoV-2 is related to its ability to survive on a variety of surfaces over the long term. ${ }^{1,2}$ At a time when the mass of viral infections means that vaccines and effective therapies against human coronaviruses are limited, reducing the density of invasive virions in the environment is gaining great medical and economic importance. The main effort of the medical services is put into the costly fight against the virus that has already infected the patient. A chance to reduce the costs is offered by technologies that will reduce the survival rate of a virus deposited on various objects. ${ }^{2}$ Infectious agents can survive from hours to days under favorable conditions. The smooth surface provides a favorable environment for the survival of the virus, but the temperature shortens its half-life. At the same time, the virus is more stable in the indoor environment with low relative humidity. ${ }^{3}$ Materials and equipment can be a source of infectious agents because virions remain active outside the host cells. Man-made substances with virucidal properties help to reduce the amount of dangerous viruses and therefore reduce the risk of infection. The SARS-CoV-2 attached on the surfaces can be inactivated through cleaning with solutions containing $0.5 \%$ hydrogen peroxide, $70 \%$ ethanol, $0.5 \%$ povidone iodine, or 0.1 sodium hypochlorite (only temporarily). ${ }^{1,4}$ To be effective, the sanitization needs periodical repetition; one should also bear in mind that it cannot be applied to every surface. ${ }^{1}$ Materials that lower the survival rate of pathogens are especially useful in medical devices. The SARS-CoV-2 coronavirus pandemic strengthens the demand for microparticles that can be combined with other materials, giving them virusinactivating properties. ${ }^{2}$

\section{Methods}

Natural antiviral active compounds acting on the SARS and MERS-CoV enzymes (nsP13 and 3CL protease helicase), for example: myricetin, scutellarein, flavonoids, tea tree oil, eucalyptus oils and phenolic compounds, are not covered in this article. ${ }^{1}$ This paper discusses the use of polymers in reducing human exposure to coronaviruses and some other viruses that cause respiratory disease. The systematic search of the PubMed database was performed in October 2020 and was focused on a part of "man-made" materials classified with indirect mechanisms of antiviral action and chemically modified materials surfaces. Research terms comprised a combination of words "polymer" and "coronavirus". The analysis included data from epidemics: SARS in 2003, MERS-CoV from 2012 and the current SARS-CoV-2.

\section{Bioinspired surfaces}

The inactivation function of antiviral agents depends on the structure of the polymer chain. The anionic nature of the polymer charge and the hydrophobicity of the backbone are key. ${ }^{5}$ Self-cleaning coatings can be applied to surfaces that are frequently touched and contaminated to avoid adhesion of infectious microdroplets. Some nanostructured, polymer-based coating materials that mimic the natural structures of lotus leaf, gecko bristles, skis, or fly eyes are superhydrophobic. ${ }^{1}$

\section{Physicochemical characteristics of antiviral polymers}

Elevated temperature in the environment increases antiviral activity of polymers. Anions of toxic ions are more effective in disinfection than nonionic metals. ${ }^{3}$ Sodium acrylonitrile/methallyl sulfonate membranes used in continuous renal replacement therapy have proven to be effective barriers to inhibit SARS-COV-2 penetration. ${ }^{6}$ Dres et al. $^{7}$ proposed a method of obtaining personal protective equipment in the form of a gluten face mask, which is a biopolymer. Gluten was supposed to be used as a filter material. The electrospinning process creates a mat of carbon nanofibers, which is used to create a filter and a laminate from which the mask is made. The polymers can be easily modified. One of the modifiable features is the electric charge which affects the quality of antivirus protection. Leung et al. improved virus capture efficiency thanks to the use of positively electrifying polyvinylidene fluoride (PVDF) nanofibers. ${ }^{8}$ Reusable medical masks (e.g., N95) must be cleaned. The use of a hydrophobic material allows partial self-cleaning. El-Atab et al. developed a method for the preparation of polyimide hydrophobic membranes with nanopores. ${ }^{9}$ Personal protective equipment (PPE) in the form of a face mask makes daily communication difficult. Face shields are less effective than face masks because they do not stick to the face. Therefore, it would be a compromise to use a transparent material with appropriate filtering properties. He et al. developed a method of producing masking filters from a nanoporous transparent polylactic acid mat with higher efficiency than standard PPE. ${ }^{10}$ Schandock et al. ${ }^{5}$ showed that polyvinylbenzoic acid (PVBzA) could be a potential antiviral agent with a wide range of applications. It has the ability to inhibit enveloped viruses ZIKV (Zika virus), HIV-1, influenza, Lyssa, Ebola and SARS. Among the polyphosphates, poly (vinylphosphonic acid) (PVPA) shows a high inhibition capacity for herpes simplex virus 2 (HSV-2) and 
Table 1. The chemical characteristics of the polymers and their role in limiting the spread of viruses

\begin{tabular}{|c|c|c|c|}
\hline Author & Code name & Type & Task \\
\hline Dres et al. & AMS & sodium acrylonitrile/methallyl sulfonate & CRRT membrane \\
\hline Schandock et al. & PVBzA & polycarboxylates & antiviral agent \\
\hline Schandock et al. & PVPA & polyphosphates & antiviral agent \\
\hline Ye et al. & GO-PVP GO-PDDA & composite & antiviral agent \\
\hline Ye et al. & GO-PDDA & composite & antiviral agent \\
\hline $\begin{array}{l}\text { Bidra et al. } \\
\text { Kariwa et al. } \\
\text { Khan et al. }\end{array}$ & PVP-I & N-vinylpyrrolidone polymer & antiviral agent \\
\hline Ahmed et al. & & metal-combined polymers & antiviral agent \\
\hline Das et al. & processed gluten & & PPE \\
\hline Leung et al. & positively electrifying PVDF & fluoropolymer & PPE \\
\hline El-Atab et al. & & polyimide & PPE \\
\hline Behzadinasab et al. & polyurethane with coper oxide $\left(\mathrm{Cu}_{2} \mathrm{O}\right)$ & composite & antiviral agent \\
\hline He et al. & nanoporous PLA & polyester & PPE \\
\hline
\end{tabular}

AMS - acrylonitrile/methallyl sulfonate; CRRT - continuous renal replacement therapy; PEG-PLGA - poly(ethylene glycol)-poly(lactide-co-glycolide); PVBzA - poly(vinylbenzoic acid); PVPA - poly(vinylphosphonic acid); PVP - poly(vinylpyrrolidone); PDDA - poly(diallyldimethylammonium) chloride; PVP-I - poly(vinylpyrrolidone)-iodine; PVDF - poly(vinylidene) fluoride; PLA - poly(lactic acid).

SARS; however, due to its very low effectiveness against other viruses, it can only be used under certain exposure conditions. These compounds can be used to form an antiviral protective layer on external surfaces. Ye et al. ${ }^{11}$ has shown that polymer-conjugated graphene oxide has antiviral properties due to the negatively charged graphene oxide (GO)-conjugated polymers. The polymers can be: non-ionic PVP (polyvinylpyrrolidone composite) and cationic PDDA (poly(diallyldimethylammonium) chloride). The inactivation mechanism is based on the cleavage of the virus by a single-layer graphene oxide. The PVP seems to be more effective.

\section{Materials containing heavy metal particles}

Some heavy metal nanoparticles have virucidal properties, hence the ideas of weaving them into the structure of various materials, including inorganic ones. Balagna et al. ${ }^{12}$ found that materials coated with silver silicate composite nanoparticles inactivate SARS-CoV-2. Also, the copper-covered surface significantly reduces the halflife of SARS-CoV-2 to $4 \mathrm{~h}$ compared to the steel-plastic surface where the virus was detectable for $72 \mathrm{~h} .{ }^{13}$ Disinfection of various items can be difficult or even impossible. The problem can be solved by using covering materials capable of inactivating the virus in production. The addition of copper to components produced on 3D printers may be helpful in increasing the virological safety of parts which, due to the method of manufacture, do not reach $100 \%$ sterility. ${ }^{14}$ Heavy metal polymers affect viral proteins and the genetic material of the virus. ${ }^{1,3}$ The cationic copper nanoparticles coupled with the polymer achieve a higher inhibitory effect than the microparticles or the metal surface. Copper ions lead to DNA denaturation, RNA damage and disturbance of viral protein synthesis. The addition of copper to PPE equipment used by healthcare professionals increases their effectiveness as a virological barrier. ${ }^{15}$ Replacing metallic copper in the polymer structure with copper oxide increases the effectiveness of PPE. Behzadinasab et al. proposed to cover some everyday items with polyurethane bound to copper oxide $\left(\mathrm{Cu}_{2} \mathrm{O}\right) \cdot{ }^{16}$ Ahmed et al. ${ }^{17}$ introduced a novel PPE filter barrier architecture, in which the polymer acts as a crosslinked mechanical barrier and scaffolding. He proposed the use of polylactic acid (PLA) and cellulose acetate (CA) to create an electrospun multilayer barrier scaffold. The nanoparticles of $\mathrm{Cu}_{2} \mathrm{O}$ and graphene oxide embedded in it inactivate viral particles that have penetrated into it.

The chemical characteristics of the polymers and their role in limiting the spread of viruses are presented in Table 1.

\section{Conclusions}

Accelerated virus inactivation polymers are a promising area of research due to the growing epidemic threat of viral diseases, in particular those caused by coronaviruses. They can make a significant contribution to slowing down the pandemic. Taking into account the different purpose of different materials and the associated physico-chemical characteristics, it seems that the future belongs to multi-component mixtures or very thin multilayer systems. The rational direction of research work is the search for materials with a balanced specificity in relation to the most dangerous viruses and universality in relation to other viruses. 


\section{ORCID iDs}

Sławomir Zmonarski (D) https://orcid.org/0000-0002-0764-4939 Jakub Stojanowski (D) https://orcid.org/0000-0002-0527-2190 Joanna Zmonarska (D) https://orcid.org/0000-0002-9678-8036

\section{References}

1. Sun Z, Ostrikov K. Future antiviral surfaces: Lessons from COVID-19 pandemic. Sustainable Materials and Technologies 2020;25:e00203e00203.

2. Zhu N, Zhang D, Wang W, et al; China Novel Coronavirus Investigating and Research Team. A novel coronavirus from patients with pneumonia in China, 2019. N Engl J Med. 2020;382(8):727-733.

3. Biryukov J, Boydston JA, Dunning RA, et al. Increasing temperature and relative humidity accelerates inactivation of SARS-CoV-2 on surfaces. mSphere 2020;5(4):e00441-e00420.

4. Khan MM, Parab SR, Paranjape M. Repurposing $0.5 \%$ povidone iodine solution in otorhinolaryngology practice in Covid 19 pandemic. Am J Otolaryngol. 2020;41(5):102618-102618.

5. Schandock F, Riber CF, Röcker A, et al. Macromolecular antiviral agents against Zika, Ebola, SARS, and other pathogenic ciruses. Adv Healthc Mater. 2017;6(23):1700748.

6. Dres M, Burrel S, Boutolleau D, et al. SARS-CoV-2 does not spread through extracorporeal membrane oxygenation or dialysis membranes. Am J Respir Crit Care Med. 2020;202(3):458-460.

7. DasO,NeisianyRE, Capezza AJ, etal.Theneedforfullybio-basedfacemasks to counter coronavirus outbreaks: A perspective. The Science of the Total Environment. 2020;736:139611-139611.

8. Leung WWF, Sun Q. Electrostatic charged nanofiber filter for filtering airborne novel coronavirus (COVID-19) and nano-aerosols. Sep Purif Technol. 2020;250:116886-116886.
9. El-Atab N, Qaiser N, Badghaish H, Shaikh SF, Hussain MM. Flexible nanoporous template for the design and development of reusable anti-COVID-19 hydrophobic face masks. ACS Nano. 2020;14(6): 7659-7665.

10. He H, Gao M, Illés B, Molnar K. 3D printed and electrospun, transparent, hierarchical polylactic acid mask nanoporous filter. Int J Bioprint. 2020;6(4):278.

11. Ye S, Shao K, Li Z, et al. Antiviral activity of graphene oxide: How sharp edged structure and charge matter. ACS Appl Mater Interfaces. 2015;7(38):21571-21579.

12. Balagna C, Perero S, Percivalle E, Nepita EV, Ferraris M. Virucidal effect against coronavirus SARS-CoV-2 of a silver nanocluster/silica composite sputtered coating. Open Ceramics. 2020;1:100006-100006.

13. van Doremalen $\mathrm{N}$, Bushmaker T, Morris $\mathrm{DH}$, et al. Aerosol and surface stability of SARS-CoV-2 as compared with SARS-CoV-1. N Engl J Med. 2020;382(16):1564-1567.

14. Kondor S, Grant CG, Liacouras P, et al. On demand additive manufacturing of a basic surgical kit. J Med Devices. 2013;7: 30916.

15. Zuniga JM, Cortes A. The role of additive manufacturing and antimicrobial polymers in the COVID-19 pandemic. Expert Rev Med Devices. 2020;17(6):477-481.

16. Behzadinasab S, Chin A, Hosseini M, Poon L, Ducker WA. A surface coating that rapidly inactivates SARS-CoV-2. ACS Appl Mater Interfaces. 2020;12:34723-34727.

17. Ahmed MK, Afifi M, Uskoković V. Protecting healthcare workers during COVID-19 pandemic with nanotechnology: A protocol for a new device from Egypt. J Infect Public Health. 2020;13(9):1243-1246. 